\title{
Duplicación de las venas gonadales, al propósito de un caso $^{33}$
}

\section{Duplication of the gonadal veins, with purpose of a case}

\author{
Euclides Dotta Neto ${ }^{34}$; Fátima Núñez; Claúdia Zanotti ${ }^{36}$
}

\section{DOI: https://doi.org/10.36003/Rev.investig.cient.tecnol.V2N2(2018)9}

\section{RESUMEN}

El varicocele es una dilatación del plexo pampiniforme, una red de venas que irrigan el testículo. Esta patología se ha relacionado con la infertilidad masculina y no discrimina edades. El varicocele ocurre por una insuficiencia en el drenaje de sangre de los testículos, que lleva al bloqueo sanguíneo o reflujo de sangre de vuelta hacia las venas del plexo pampiniforme y aumenta el volumen de las venas. En el estudio se relaciona el varicocele con la duplicación de las venas gonadales izquierdas que desembocan en la vena renal izquierda. Las venas gonadales, también conocidas como venas testiculares en hombres y venas ováricas en mujeres pueden presentar anomalías. Normalmente, en una persona solo una vena desemboca en la vena renal izquierda, pero en ese caso de anomalía difiere por la vena gonadal duplicarse y drenar directamente en la vena renal izquierda. Estas anomalías pueden ser debido a factores estructurales, funcionales, metabólicos o displasia. Se confirmó que en las personas que tienen anomalías de venas gonadales izquierdas, puede haber un riesgo mayor de tener varicocele, debido a la distancia que recorre la sangre hasta a la vena renal, la presión para empujar la sangre, defectos de las válvulas, insuficiencia valvular, el espesor de la vena o también el ángulo de recorrido sanguíneo, donde ocurre una disminución de la presión en las áreas bajas del sistema, favoreciendo el aparecimiento de tortuosidades del plexo pampiniforme, que se llenan para aumentar la presión y soportar la fuerza de la gravedad.

Palabras clave: Anatomía macroscópica; Anomalía congénita; Duplicación de venas; Varicocele; Venas gonadales.

\footnotetext{
${ }^{33}$ Fecha de recepción: septiembre 2018; fecha de aceptación: octubre 2018

${ }^{34}$ Estudiante de la Carrera de Medicina de la Universidad Privada María Serrana, Asunción, Paraguay.

${ }^{35}$ Profesoras de la Cátedra de Anatomía II de la Universidad Privada María Serrana, Asunción, Paraguay
} 


\section{ABSTRACT}

Varicocele is a dilatation of the pampiniform plexus, one set of veins that drains the testicle. This pathology has been related to male infertility and it does not discriminate ages. The Varicocele happens due to insufficient drainage of blood from the testicles, which takes to blood blockage or reflux of blood back into the veins of pampiniform plexus and increase in the volume of the veins. The study relates the varicocele with duplication of left gonadal veins that drains into the left renal vein. Gonadal veins, also known as testicular veins in men and ovarian veins in women, can present anomalies. Normally, in a person only one vein drain into left renal vein, but in this case of anomaly it differs by double veins and it drains directly into left renal vein. These anomalies may be due to structural factors, functional factors, metabolic factors or dysplasia. It was confirmed that in people who have anomalies of left gonadal veins, there may be a greater risk of having varicocele, due to the distance that runs blood to the renal vein, pressure to push the blood, valves defects, valvular insufficiency, vein thickness or blood travel angle as well, where there is a decrease in pressure in the lower areas of the system, favoring the appearance of tortuosity of the pampiniform plexus, that are filled to increase pressure and endure the force of gravity.

Keywords: Macroscopic anatomy; Congenital anomaly; Vein duplication; Varicocele; Gonadal veins 


\section{INTRODUCCIÓN}

En el sistema circulatorio, la vena es responsable del transporte de la sangre hasta el corazón. En nuestro cuerpo hay dos tipos de circulación de venas así como de las arterias, de acuerdo con Rouviere, los cuerpos poseen venas de circulación menor o circulación pulmonar y venas de circulación mayor o circulación sistémica (1).

Las venas gonadales en hombres son llamadas venas testiculares y en las mujeres venas ováricas. Las venas testiculares y ováricas siguen el mismo trayecto retroperitoneal de las arterias.

En los hombres las venas testiculares proceden del testículo y epidídimo, donde se unen y forman una red venosa o plexo pampiniforme, compuesto de 10 a 15 vénulas sinuosas, que ascienden y se anastomosan, disminuyendo progresivamente de número (1).

Moore, señala que las venas de cada plexo pampiniforme confluyen en la parte superior para formar la vena testicular derecha que desemboca en la vena cava inferior (VCI) y la vena testicular izquierda que termina en la vena renal izquierda (2).

Canby, considera que la funcionalidad de los plexos pampiniforme es básicamente disminuir la temperatura testicular (3).

En las mujeres, las venas que drenan en el ovario forman también un plexo pampiniforme como al de los hombres, pero en forma de enredadera en el ligamento ancho próximo al ovario y la trompa uterina (2).

Las venas se anastomosan en el plexo venoso pampiniforme, ascienden a lo largo de la arteria, se reduce a dos troncos que se unen en un sola vena ovárica (1).

Las venas ováricas izquierdas se unen a la vena renal izquierda y las venas ováricas derechas lo hacen a la vena cava inferior en el abdomen (4).

Las anomalías congénitas son malformaciones del desarrollo presentes en el naci- miento. Un bebe puede nacer saludable o presentar alguna anomalía congénita, que puede ser causada por un problema genético o inducida por usos de sustancias nocivas durante la gestación, o también por la falta de líquido amniótico (5).

Estas anomalías pueden ser por factores estructurales, funcionales, metabólicos o displasia.

El varicocele se caracteriza por una dilatación o tortuosidad del plexo pampiniforme, una red de venas que irrigan los testículos (3).

Los vasos sanguíneos que nutren al testículo se originan en el abdomen y descienden por el conducto inguinal, formando parte del cordón espermático, hasta llegar a la gónada. El flujo de sangre venoso controlado por válvulas impide el reflujo sanguíneo, cuando ocurre un defecto en esas válvulas o la compresión de las venas por estructuras adyacentes, pueden causar la dilatación de estas venas y originar un varicocele (6).

El varicocele ocurre por una insuficiencia en el drenaje de sangre del testículo, que lleva al represamiento sanguíneo y al aumento del volumen de las venas.

Esta patología se ha relacionado con la infertilidad masculina, disminución en el número de espermatozoides, de su movilidad, morfología y capacidad de fecundación (7).

En las mujeres se localizan en las venas ováricas y favorece el que aparezcan varices pélvicas y en las piernas, favoreciendo la llamada congestión pelviana (6).

El varicocele afecta todas las edades: $7,2 \%$ en los individuos de 2 a 19 años, después de los 20 años la porcentaje es de $10 \%$ a $25 \%$ y en individuos adultos el varicocele está presente hasta en 42,9\% (8).

La incidencia del varicocele izquierdo es superior a la del derecho. En 504 casos, se obtuvo $85,5 \%$ de varicoceles izquierdos, $14 \%$ bilaterales y un $0,4 \%$ derechos (9). 
La diferencia entre la incidencia de la izquierda y derecha se debe a las bases anatómicas: mayor longitud de la vena testicular izquierda que la derecha; menor frecuencia en las válvulas venosas en el lado izquierdo; mayor frecuencia del número de válvulas ostiales competentes en el lado derecho que en el izquierdo; desemboca en un ángulo recto o sea de aproximadamente $90^{\circ}$ de la vena testicular en la vena renal, haciéndolo en un ángulo de $30^{\circ}$ a $40^{\circ}$ en la derecha; posibilidad de pinzar la vena renal izquierda por la horquilla aortomesentérica (9).

Generalmente, el tratamiento del varicocele puede ser de dos maneras: 1) Procedimiento microquirúrgico o ligadura quirúrgica de las venas varicosas, en este procedimiento se usa al microscopio para percibir mejor las venas y otras áreas de la ingle y se realiza la ligadura de los vasos alterados o amarre de las estructuras. 2) Embolización percutánea que es un procedimiento no quirúrgico, donde se realiza una pequeña incisión en la ingle. Por esa incisión, se inserta un catéter en la vena femoral (10).

El estudio se realizó en el laboratorio de anatomía de la Universidad Privada María Serrana, se observó un caso de duplicación parcial de la vena gonadal en un cadáver como unidad de análisis.

Los materiales utilizados fueron: un algodón para sacar afuera las grasas alrededor de las venas con propósito de mejorar las imágenes; una regla para hacer las mediciones de las venas y una pinza para facilitar el trabajo de manejar las venas.

Se plantearon las siguientes interrogantes de investigación ¿Por qué las personas que tienen anomalías en las venas gonadales, tienen una predisposición mayor de tener varicocele? ¿Por qué el varicocele afecta más en el lado izquierdo comparado al derecho?

El objetivo general fue analizar la dupli- cación de las venas gonadales con la patología de varicocele.

En la Figura 1, puede notarse la duplicación parcial de las venas; las dos venas gonadales empiezan juntas en la parte inferior, después, ocurre una bifurcación, generando dos venas independientes, las venas representadas por el número 1 y 2 de la imagen, drenan directo a la vena renal izquierda. En la vena gonadal lateral, denominada con el número 2 , se nota una ramificación próxima a la vena renal izquierda, teniendo $5 \mathrm{~mm}$ de longitud, identificada con el número 3 .

El número 1 en la imagen representa la vena gonadal izquierda "medial" en ese caso de bifurcación; el número 2 es la vena gonadal izquierda "lateral" y el número 3, rama colateral de la vena izquierda "lateral", que también drena a la vena renal izquierda.

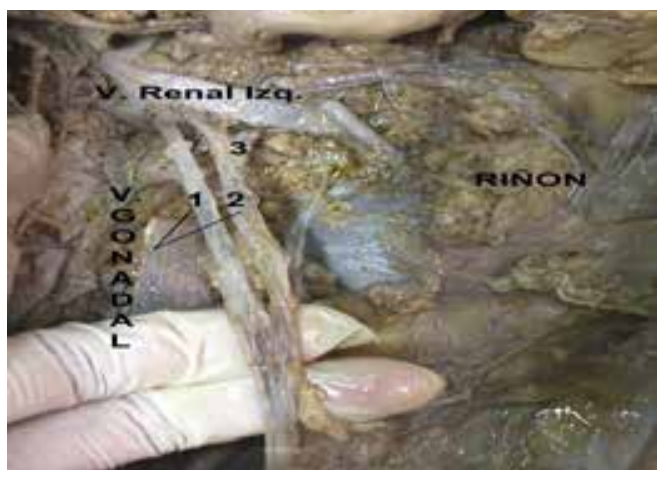

Figura 1. Duplicación de las venas gonadales. 
En la Figura 2, la vena gonadal medial, representada por el número 1 y la lateral representada por el número 2 , tienen una medida de 4,5 $\mathrm{mm}$ de ancho, y el número 4 muestra una distancia entre ellos de $2 \mathrm{~mm}$. La tercera vena o también denominada rama colateral de la vena gonadal lateral representada por el número 3 , tiene $4 \mathrm{~mm}$ de ancho. La distancia de la rama colateral hasta el margen medial del riñón es de $26 \mathrm{~mm}$ (número 9), la vena lateral tiene 39 $\mathrm{mm}$ de distancia (número 8 ) y la más distal o medial tiene una distancia de $46 \mathrm{~mm}$ (número 7). Desde a bifurcación hasta la vena renal izquierda, hay un camino de 76 $\mathrm{mm}$ de longitud, como muestra el número 5 en la figura 2).

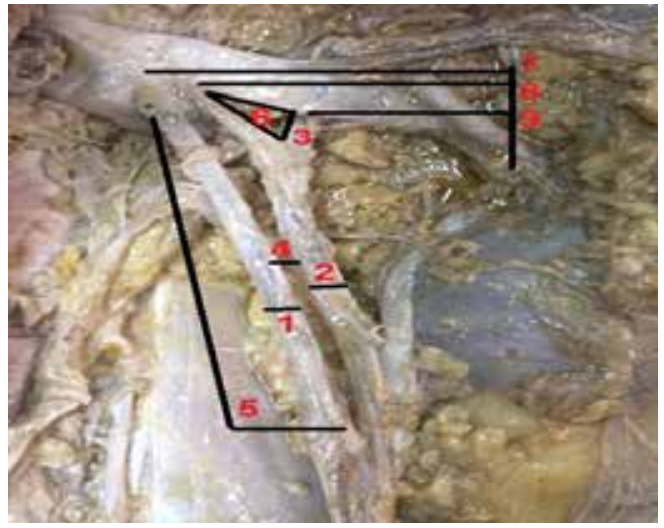

Figura 2: Esquema de duplicación de la vena gonadal
La bifurcación de las venas 2 y 3 forman un triángulo que posee un ángulo en la base lateral representado por la letra $B$ de $49^{\circ}$ y distancia $\mathrm{B}$ hasta $\mathrm{A}$ es de $6 \mathrm{~mm}$; el ángulo de $\mathrm{A}$ es de $110^{\circ}$ y su distancia hasta $C$ es de $9 \mathrm{~mm}$; finalmente, para cerrar el triángulo tenemos $21^{\circ}$ en la punta $\mathrm{C}$ y su distancia hasta $\mathrm{B}$ es de $10 \mathrm{~mm}$. (Fig. 3).

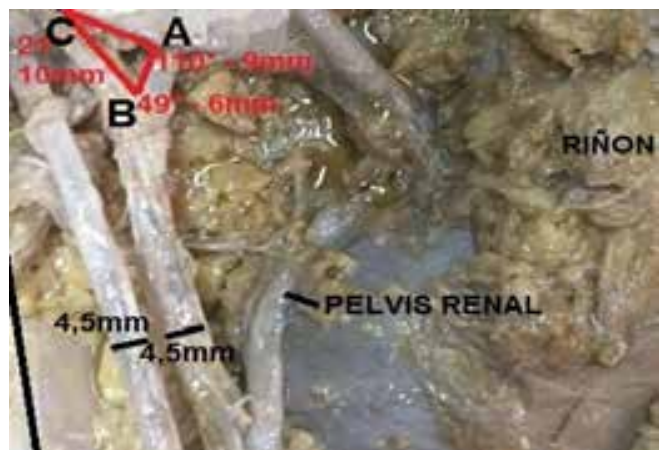

Figura 3: Triángulo formado por las venas gonadales 2 y 3 


\section{DISCUSIÓN}

De acuerdo con Asala, quien examinó 150 cadáveres, expresó que las anomalías eran más frecuentes en las venas gonadales que en las arterias acompañantes y también en la mayoría de los casos ocurre en la parte izquierda del cuerpo humano. Identificó anomalías en 32 de los 150 cadáveres, obteniendo un 21,3\%. Las venas se presentaban completa o parcialmente duplicadas. Las venas duplicadas terminaban en la vena renal izquierda separadamente o también, combinado en otra vena (11).

La incidencia de varicocele en la población general se reporta entre 10 a $15 \%$, en pacientes la incidencia de infertilidad primaria se encuentra entre 30 a $50 \%$ y pacientes con infertilidad secundaria es de $80 \%$. El varicocele en la adolescencia se reporta entre 16 a $28 \%$ y en adultos la incidencia es del 42 a 75\% (7).

Consecuentemente, la anomalía presentada en las venas gonadales puede derivarse de cambios en el origen embriológico de esas venas.

Existen múltiples anomalías congénitas, la mayoría de ellas sin trascendencia clínica, pero de gran importancia quirúrgica. Es importante que los médicos identifiquen las variantes anatómicas del aporte vascular habitual, ya que no hacerlo genera complicaciones quirúrgicas e impide ciertas intervenciones terapéuticas (12).

El varicocele izquierdo se describe con mayor ocurrencia en la parte izquierda, donde el curso sanguíneo termina en la vena renal, siguiendo media de 15 a 20 $\mathrm{cm}$ contra la gravedad en un ángulo de casi $90^{\circ}$, dificultando el flujo del torrente sanguíneo, mientras en la vena derecha ese camino es diferente, donde la vena gonadal derecha desemboca oblicuamente en la vena cava, facilitando la drenaje de la sangre, el camino es más corto y presenta un ángulo de $30^{\circ}$ a $40^{\circ}$.
Por lo tanto es mayor la tendencia de ocurrir una insuficiencia en el drenaje de sangre del testículo que lleva al bloqueo sanguíneo, y hace que el volumen de las venas aumente y así genere el varicocele, considerando la dificultad para que la sangre llegue hasta la vena renal.

Con este estudio de caso, fue posible confirmar que en las personas que tienen anomalías de venas gonadales izquierdas, puede haber un riesgo mayor de tener varicocele en la parte izquierda; ambas ocurren con más frecuencia en la parte izquierda que en la derecha del cuerpo humano.

Se puede notar una coincidencia, donde ambas, tanto la anomalía con la patología, generalmente ocurre más del lado izquierdo que del derecho en el cuerpo humano, pudiendo asociarse a la distancia que recorre la sangre hasta a la vena renal, la presión para empujar la sangre hacia arriba, defectos de las válvulas, insuficiencia valvular, el espesor de la vena o también el ángulo de recorrido sanguíneo.

Cuando hay una anomalía como la duplicación de la vena, esta vena necesita de una doble fuerza, para empujar la sangre hasta llegar a la vena renal, ocurre una disminución de la presión en las áreas bajas del sistema, favoreciendo el aparecimiento de tortuosidades del plexo pampiniforme, que en consecuencia, se llenan para aumentar la presión y soportar la fuerza de la gravedad.

Sin embargo, esta insuficiencia en el drenaje de sangre, puede hacer que ocurra un bloqueo sanguíneo o reflujo de sangre de vuelta hacia las venas del plexo pampiniforme y aumento del volumen de las venas, desarrollándose un varicocele. 


\section{BIBLIOGRAFÍA}

1. ROUVIERE, H. \& DELMAS, A. Anatomía Humana. Descriptiva, Topográfica y Funcional. Tomo 3. 11 ed. Barcelona, Masson, 2005. 231-243p.

2. MOORE, K.L. Anatomía con Orientación Clínica. $5^{\mathrm{a} e d}$. Wolkers Kluwer, 2008. 228-427p.

3. CANBY, C.A. Anatomía Basada en la Resolución de Problemas. Elsevier. Sección IV. Case 34, 2006. Disponible en $<$ https://books.google.com.py/ > Consultado el 28 de Octubre de 2018.

4. DRAKE, R.L. et al. Gray: Anatomia Para Estudiantes. $1^{\mathrm{a}}$ ed. Elsevier, 2006. 432p.

5. MARTINS, ELISA, 2018. Anomalías Congénitas. Disponible en $<$ https://www.infoescola.com/genetica/anomalias-congenitas/ $>$. Consulta el 12 de Octubre de 2018.

6. SEMI - SOCIEDAD ESPAÑOLA DE MEDICINA INTERNA. Varicocele - ¿ En Qué Consiste Esta Enfermedad?. Disponible en < https://www.fesemi.org/ informacion-pacientes/conozca-mejor-suenfermedad/varicocele $>$. Consulta el 31 de Octubre de 2018.
7. VASQUÉZ, D. et al. Varicocele Testicular en Adolescentes. Revista Científica Salud Uninorte, Vol. 25, n. 2, Julio-Diciembre de 2009.

8. SADI, M. et al. Varicocele. [s.1.]: Associação Médica Brasileira e Conselho federal de Medicina, 2008.

9. DELGADO MARTÍN, J.A. Fisiopatología del Varicocele. Clínicas Urológicas de la Complutense, I. Ed. Complutense, Madrid, 1992. 393p.

10. InSTITUTO GERA. O Que é Variocele ? Como é o Tratamento ?. Disponible en $<$ https://clinicagera. com.br/pergunta/o-que-e-varicocele-como-e-o-tratamento/ $>$. Consulta el 31 de Octubre de 2018.

11. ASALA, S. et al. Anatomical Variations in the Human Testicular Blood Vessels. Annals of Anatomy, v.183, issue 6. 2001. 545-549p.

12. ROBBINS Y COTRAN. Anomalías Vasculares. Patología Estructural y Funcional, 9 Ed. Elsevier Saunders, España, 2015. 485p. 